\title{
Artificial intelligence (AI) and interventional radiotherapy (brachytherapy): state of art and future perspectives
}

\author{
Bruno Fionda, MD!, Luca Boldrini, MD!,2, Andrea D'Aviero, MD², Valentina Lancellotta, MD! \\ Maria Antonietta Gambacorta, MD, PhDl,2, György Kovács, MD, PhD³, Stefano Patarnello, Physicist!, \\ Vincenzo Valentini, MD',2, Luca Tagliaferri, MD, PhD \\ IUOC Radioterapia Oncologica, Dipartimento di Diagnostica per Immagini, Radioterapia Oncologica ed Ematologia, Fondazione Policlinico \\ Universitario A. Gemelli, IRCCS, Roma, Italy, ${ }^{2}$ Università Cattolica del Sacro Cuore, Roma, Italy, ${ }^{3}$ Universitò Cattolica del Sacro Cuore, \\ Educational Program Director Gemelli-INTERACTS, Roma, Italy
}

\begin{abstract}
Purpose: Artificial intelligence (AI) plays a central role in building decision supporting systems (DSS), and its application in healthcare is rapidly increasing. The aim of this study was to define the role of AI in healthcare, with main focus on radiation oncology (RO) and interventional radiotherapy (IRT, brachytherapy).

Artificial intelligence in interventional radiation therapy: $\mathrm{AI}$ in $\mathrm{RO}$ has a large impact in providing clinical decision support, data mining and advanced imaging analysis, automating repetitive tasks, optimizing time, and modelling patients and physicians' behaviors in heterogeneous contexts. Implementing AI and automation in RO and IRT can successfully facilitate all the steps of treatment workflow, such as patient consultation, target volume delineation, treatment planning, and treatment delivery.

Conclusions: AI may contribute to improve clinical outcomes through the application of predictive models and DSS optimization. This approach could lead to reducing time-consuming repetitive tasks, healthcare costs, and improving treatment quality assurance and patient's assistance in IRT.

Key words: artificial intelligence, interventional radiotherapy, brachytherapy, personalized medicine, machine learning, decision supporting system.

\section{Purpose}

Radiation oncology (RO) family sees three main areas of application based on the modality of tumor treatments, including external beam radiotherapy (EBRT), through the use of sophisticated machines that produce radiation away from the body; metabolic radiotherapy (MRT) involving the use of radiopharmaceuticals that yield therapeutic results by the direct interaction to cells metabolism; and finally, interventional radiotherapy (IRT, brachytherapy). In IRT, with the use of applicators and catheters (therapeutic implants) placed in contact or inside the tumor, one or more sealed radioactive sources are placed to destroy malignant cells. The main feature of this "internal" radiation mode is the ability to deliver high radiation doses to the target tissue while sparing the surrounding organs due to steep dose falloff from the source. The availability of miniaturized radioactive sources and new devices, which facilitated the radiation protection of patients and staff, the integration with modern imaging for better definition of the target volume, the correct positioning of sources/applicators, and the possibility of modulating the intensity of dose point-by-point (intensity modulated interventional radiotherapy), have fostered a new interest in the modern use of this application technique.

In the scenario of healthcare, precision medicine (PM) is emerging both in the context of preventing and treating diseases. More specifically, PM has significant applications in cancer care, identifying specific patient populations at risk and developing alternative and more accurate treatment options based on the evaluation of individual variability [1]. PM is characterized by four features: it is predictive, preventive, personalized, and participatory. The analysis of individual genomic and biological pathways results in a more accurate definition of clinical outcomes related to a certain disease and treatment, leading to the increase of predictivity and the adoption of preventive measures. 
Moreover, personalization is a major aspect of applying PM in oncology heading from "average-population"based therapeutic choices to subpopulations' models. The participatory effort of different figures is crucial, in the frame of a personalized therapeutic/diagnostic approach; establishing large networks of reference centers and big data collection can create definitive decision support systems (DSS) to share the data and assist clinicians in decision-making.

Artificial intelligence (AI) computational methods allow to perform tasks that typically require human intelligence by extracting rules and "learn" from data while processing them. The use of machine learning (ML) to replicate and predict human behavior and mental skills in learning and decision-making, its application in healthcare, and personalized medicine is rapidly increasing through the definition of specific algorithms [2].

One important field for application of AI in healthcare is radiotherapy (RT). Because of the results in terms of tumor control in radical setting, but also due to the effective and durable palliation and pain control, this treatment provides a high degree of personalization (i.e., therapy volume and dose selection) among the different cancer therapies. It is involved in $45-55 \%$ of newly diagnosed cancers and even in the advanced stage diseases [3]. However, RT requires high training standards and quality assurance, as it is a technologically complex and advanced treatment approach, the adoption of $\mathrm{AI}$ methods can be highly beneficial to improve quality of treatment and overall effectiveness.

The aim of this review was to assess current status and future perspectives in the field of AI, defining its application in RO, with a particular focus on IRT (brachytherapy).

\section{Role of artificial intelligence in radiation oncology}

The introduction of automation and AI could be considered when pursuing quality assurance in clinical prac- tice. The impact of $\mathrm{AI}$ in $\mathrm{RO}$, in terms of ML, deep learning, big data, and data modelling innovation [4], may result in short-term overall health quality improvement.

Artificial intelligence, as a part of automation, has been systematically involved in radiation treatment workflow [5,6]. As a first example, in most recent years, several commercial auto-segmentation solutions have been released with the aim to provide reliable and fast segmentations, and paving the way to perform only adaptive re-planning procedures $[7,8]$.

Many AI applications have also been introduced in RO. Several technologies can help to provide DSS tools based on large database processing, data mining, and advanced quantitative imaging analysis $[9,10,11]$. The adoption of these tools improve clinical practice through the development of predictive models, which consider numerous different decisional parameters, such as patient's comorbidities [12], and reduce the knowledge gaps between domain-specific experts and non-experts, successfully enhancing PM in oncology fields.

The use of $\mathrm{AI}$ in $\mathrm{RO}$ can also result in facilitating repetitive tasks (i.e., various treatment planning procedures) and optimizing/gaining time for clinicians to focus on modelling behaviors in heterogeneous contexts as well as enforce interaction with patients. Automation seems also to provide a significant reduction in care costs [13].

The following characteristics can be applied in all phases of radiation treatments workflow (Figure 1):

- First patient consultation: The application of AI in this step could result in improving patient-tailored treatment personalization, guiding clinical choices and treatment selection, comparing several kind of treatments [14], and thoroughly informing the patient on treatment tolerance and expected toxicities $[15,16]$.

- Delineation: Implementation of AI in this process may result in time saving and workflow efficiency optimization, with better accuracy, oncology standardization, and adequate segmentation guidelines.
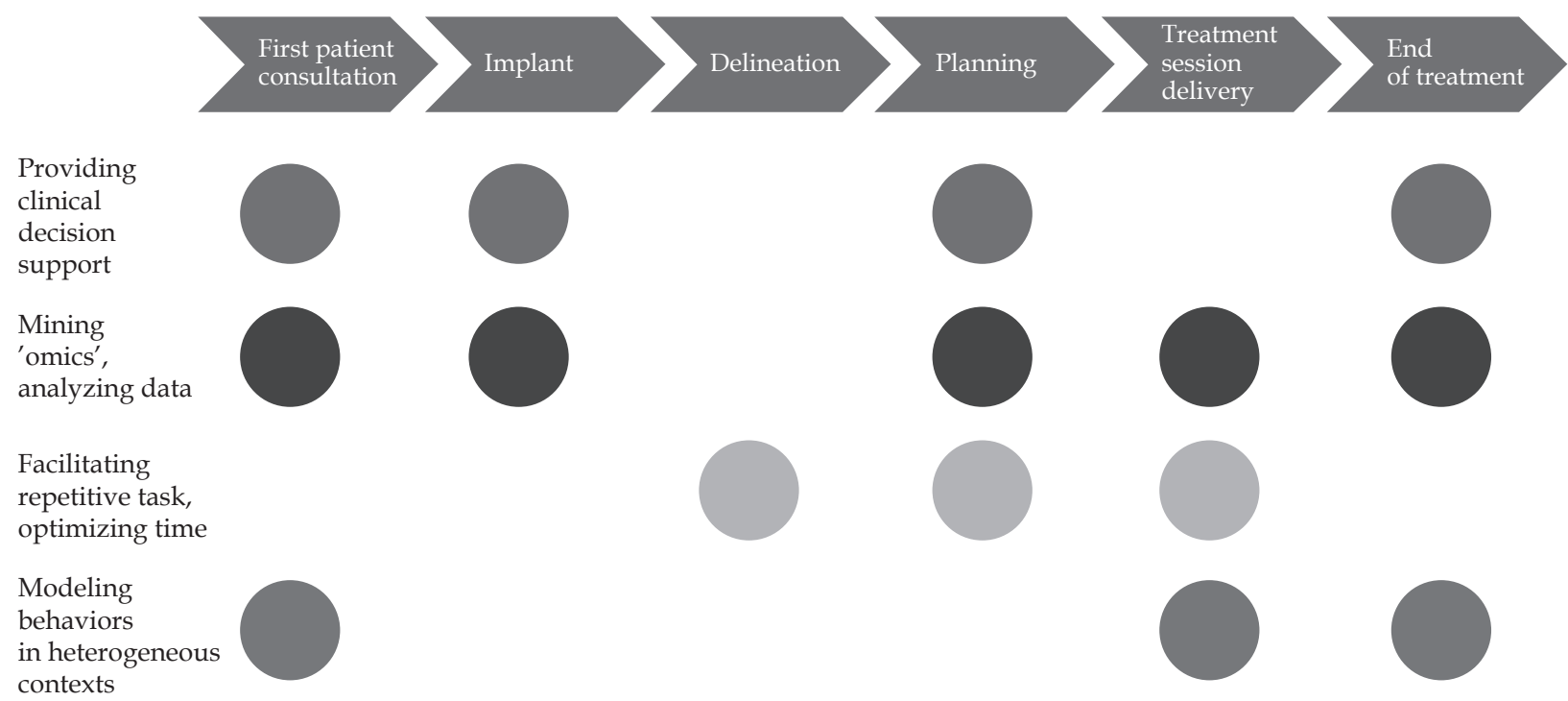

Fig. 1. Artificial intelligence in interventional radiation oncology 
- Planning: Improvement in speed and quality of a planning process might be achieved through inverse planning optimization and automated knowledge-based treatment planning approaches, potentially offering plans comparisons using different delivery technologies (i.e., photons vs. protons, or external beam vs. IRT) [17].

- Treatment session delivery: Motion management technologies for both target volumes and organs at risk (OARs) [18] could speed-up patient's repositioning [7], and improve the accuracy of treatment delivery, reducing planning margins and unnecessary irradiation.

- End of treatment: Understanding patients' needs and establishing adequate post-treatment monitoring and rehabilitation protocols, represent the main objectives of AI implementation in this step. Patients' quality of life and perception of care actions could be significantly improved thanks to AI applications.

\section{Role of artificial intelligence in interventional radiation therapy}

In the last decade, due to technology improvements, IRT has reached a rapid evolution in terms of implants, planning, and delivery $[19,20]$. These technological advancements required specific training for physicians, but it led to the increase of IRT treatments quality. Such innovations, together with the widespread use of 3D imaging techniques, including computed tomography (CT), magnetic resonance imaging (MRI), positron emission tomography (PET/CT), and ultrasound, have determined the emergence of image-guided interventional radiotherapy (IG-IRT), with important clinical benefits [21]. The development of equipment with miniaturized treatment sources (remote afterloading high-dose-rate [HDR] and pulsed-dose-rate [PDR] devices), and progressively smaller, manageable, and CT/MR compatible applicators, provided a significant contribution to this evolution [20]. The diffusion of single-source devices allowed the implementation of treatment plans with the possibility of an optimization in the coverage of the target by limiting the dose to organs at risk (intensity-modulated IRT) [22]. This led to an increase in treatment accuracy and to the development of complex image-based control systems as well as stability of plans and quality assurance protocols [23]. Potential role of artificial intelligence in IRT (Table 1) has been investigated in the last two decades.

Implant geometry and the choice of appropriate applicators is a crucial phase of IRT treatment workflow. Artificial intelligence implementation could result in providing clinical decision support, and mining 'omics' and analyzing data (Figure 1).

Several studies have underlined that AI could help to optimize the applicators location in treatment planning phases as well as the importance of predicting the optimal source position in targets, avoiding radiation of organs at risk (OARs) at best in the field of low-dose-rate (LDR) IRT $[24,25,26,27]$. Thanks to machine learning analysis of pre- and post-plan seeds disposition, effective algorithms were developed in order to obtain adequate target coverage and optimal OARs avoidance [28,29]. Intra-fractional
Table 1. Fields of application of Al in interventional radiotherapy

\begin{tabular}{lcc} 
& \multicolumn{1}{c}{ Authors } & Year \\
\hline Optimize applicators' & Yu Y et al. [24] & 1999 \\
location & Miller S et al. [25] & 2001 \\
& Yao L et al. [26] & 2015 \\
& Jiang Y et al. [27] & 2018 \\
\hline Treatment planning & Al-Qaisieh B et al. [28] & 2003 \\
and delivery & Leydon P et al. [29] & 2015 \\
& Jaberi R et al. [30] & 2017 \\
& Nicolae A et al. [31] & 2020 \\
\hline Predicting treatment & Valdes G et al. [32] & 2018 \\
response & &
\end{tabular}

dose variations could result in higher toxicities and delivery uncertainties; AI models may be able to optimize planning and motion management, achieving a more safe treatment delivery [30]. Recently, initial results from a phase I trial study on prostate cancer and LDR IRT have been released [31]. Also, ML was applied to the prediction of treatment response for biochemical failure following prostate salvage HDR IRT [32]. A multicenter storage of pretreatment and delivery imaging to correlate to data of clinical outcomes could contribute to the definition of predictive models of response to treatment, with the creation of support tools to improve the positioning of catheters and the creation of treatment plans [33].

In particular, a potential field of investigation could be the integration and implementation of imaging fusion and neuro-navigation systems in clinical practice [34]. New trials are currently ongoing in order to provide new data on the applications of AI in HDR IRT $[35,36]$.

\section{Conclusions}

Artificial intelligence has a significant impact in all the steps of IRT process. Due to human intervention and automatic error detection systems, AI could also provide a reduction of cancer care costs and time-consuming repetitive tasks. Implementing AI in IRT could result in significant advantages for physicians, allowing more time for interaction with patients, increasing the level of humanity, and patients care quality.

\section{Disclosure}

All authors disclose some financial and personal relationships with other people or organizations, even if not directly relate to the submitted work.

\section{References}

1. Vineis P, Wild CP. The science of precision prevention of cancer. Lancet Oncol 2017; 18: 997-998.

2. Jarrett D, Stride E, Vallis K, Gooding MJ. Applications and limitations of machine learning in radiation oncology. $\mathrm{Br} \mathrm{J} \mathrm{Ra}$ diol 2019; 92: 20190001.

3. Tagliaferri L, Vavassori A, Lancellotta V et al. Can brachytherapy be properly considered in the clinical practice? Trilogy project: The vision of the AIRO (Italian Association of 
Radiotherapy and Clinical Oncology) Interventional Radiotherapy study group. J Contemp Brachytherapy 2020; 12: 84-89.

4. Xing L, Krupinski EA, Cai J. Artificial intelligence will soon change the landscape of medical physics research and practice. Med Phys 2018; 45: 1791-1793.

5. Thompson RF, Valdes G, Fuller CD et al. The future of artificial intelligence in radiation oncology. Int J Radiat Oncol Biol Phys 2018; 102: 247-248.

6. Boldrini L, Bibault JE, Masciocchi C et al. Deep learning: a review for the radiation oncologist. Front Oncol 2019; 9: 977.

7. Montanaro T, Nguyen DT, Keall PJ et al. A comparison of gantry-mounted x-ray-based real-Time target tracking methods. Med Phys 2018; 45: 1222-1232.

8. Valentini V, Boldrini L, Damiani A, Muren LP. Recommendations on how to establish evidence from auto-segmentation software in radiotherapy. Radiother Oncol 2014; 112: 317-320.

9. Arimura H, Soufi M, Kamezawa $\mathrm{H}$ et al. Radiomics with artificial intelligence for precision medicine in radiation therapy. J Radiat Res 2019; 60: 150-157.

10. Dinapoli N, Alitto AR, Vallati M et al. RadioBio data: A moddicom module to predict tumor control probability and normal tissue complication probability in radiotherapy. Available from: https://github.com/kbolab/moddicom.git (cited 2020 Apr 7).

11. Dinapoli N, Alitto AR, Vallati M et al. Moddicom: A complete and easily accessible library for prognostic evaluations relying on image features. Annu Int Conf IEEE Eng Med Biol Soc 2015; 2015: 771-774.

12. Ferrandina G, Lucidi A, Paglia A et al. Role of comorbidities in locally advanced cervical cancer patients administered preoperative chemoradiation: Impact on outcome and treatment-related complications. Eur I Surg Oncol 2012; 38: 238-244.

13. Massaccesi M, Corti M, Azario L et al. Can automation in radiotherapy reduce costs? Acta Oncol 2015; 54: 1282-1288.

14. Frakulli R, Galuppi A, Cammelli S et al. Brachytherapy in non melanoma skin cancer of eyelid: A systematic review. J Contemp Brachytherapy 2015; 7: 497-502.

15. Brown SA. Principles for developing patient avatars in precision and systems medicine. Front Genet 2016; 6: 365 .

16. Pagliara MM, Tagliaferri L, Lenkowicz J et al. AVATAR: analysis for visual acuity prediction after eye interventional radiotherapy. In Vivo 2020; 34: 381-387.

17. Nwankwo O, Mekdash H, Sihono DSK et al. Knowledgebased radiation therapy (KBRT) treatment planning versus planning by experts: Validation of a KBRT algorithm for prostate cancer treatment planning. Radiat Oncol 2015; 10: 111.

18. Antico M, Prinsen P, Cellini F et al. Real-time adaptive planning method for radiotherapy treatment delivery for prostate cancer patients, based on a library of plans accounting for possible anatomy configuration changes. PLoS One 2019; 14: e0213002.

19. Han K, Milosevic M, Fyles A et al. Trends in the utilization of brachytherapy in cervical cancer in the United States. Int J Radiat Oncol Biol Phys 2013; 87: 111-119.

20. Hoskin PJ, Bownes P. Innovative technologies in radiation therapy: brachytherapy. Semin Radiat Oncol 2006; 16: 209-217.

21. Haie-Meder C, Siebert FA, Pötter R. Image guided, adaptive, accelerated, high dose brachytherapy as model for advanced small volume radiotherapy. Radiother Oncol 2011; 100: 333-343.

22. Niehoff P, Dietrich J, Ostertag H et al. High-dose-rate (HDR) or pulsed-dose-rate (PDR) perioperative interstitial intensity-modulated brachytherapy (IMBT) for local recurrences of previously irradiated breast or thoracic wall following breast cancer. Strahlenther Onkol 2006; 182: 102-107.

23. Williamson JF. Current brachytherapy quality assurance guidance: does it meet the challenges of emerging image- guided technologies? Int J Radiat Oncol Biol Phys 2008; 71 (1 Suppl): S18-22.

24. Yu Y, Anderson LL, Li Z et al. Permanent prostate seed implant brachytherapy: Report of the American Association of Physicists in Medicine Task Group No. 64. Med Phys 1999; 26: 2054-2076.

25. Miller S, Bews J, Kinsner W. Brachytherapy cancer treatment optimization using simulated annealing and artificial neural networks. Can Conf Electr Comput Eng 2001; 1: 649-654.

26. Yao L, Wang J, Jiang $Y$ et al. Permanent interstitial 125 I seed implantation as a salvage therapy for pediatric recurrent or metastatic soft tissue sarcoma after multidisciplinary treatment. World J Surg Oncol 2015; 49: 1-9. Available from: http:/ / dx.doi.org/10.1186/s12957-015-0747-7

27. Jiang Y, Ji Z, Guo F et al. Side effects of CT-guided implantation of I seeds for recurrent malignant tumors of the head and neck assisted by 3D printing non co-planar template. Radiat Oncol 2018; 13: 18.

28. Al-Qaisieh B, UK Prostrat Brachytherapy Group. Pre- and post-implant dosimetry: An inter-comparison between UK prostate brachytherapy centres. Radiother Oncol 2003; 66: 181-183.

29. Leydon P, Sullivan F, Jamaluddin F. Machine learning in prediction of prostate brachytherapy rectal dose classes at day 30 . In: Proceedings of the 17th Irish Machine Vision and Image Processing Conference, 2015, pp. 105-109.

30. Jaberi R, Siavashpour Z, Aghamiri MR et al. Artificial neural network based gynaecological image-guided adaptive brachytherapy treatment planning correction of intra-fractional organs at risk dose variation. J Contemp Brachytherapy 2017; 9: 508-518.

31. Nicolae A, Semple M, Lu L et al. Conventional vs machine learning-based treatment planning in prostate brachytherapy: results of a phase I randomized controlled trial. Brachytherapy 2020; 19: 470-476.

32. Valdes G, Chang AJ, Interian $Y$ et al. Salvage HDR brachytherapy: multiple hypothesis testing versus machine learning analysis. Int J Radiat Oncol Biol Phys 2018; 101: 694-703.

33. Valentini V, Dinapoli N, Damiani A. The future of predictive models in radiation oncology: from extensive data mining to reliable modeling of the results. Future Oncol 2013; 9: 311-313.

34. Gao Y, Han Y, Nan G et al. Value of CT-MRI fusion in iodine-125 brachytherapy for high-grade glioma. Oncotarget 2017; 8: 112883-112892

35. Study on adaptive radiotherapy and multimodal information of cervical cancer assisted by artificial intelligence. ClinicalTrials.gov [Internet]. Available from: https://clinicaltrials.gov/ct2/show/NCT04022018 (cited 2020 Apr 7).

36. Machine-learning Optimization for Prostate Brachytherapy Planning (MOPP). ClinicalTrials.gov [Internet]. Available from: https://clinicaltrials.gov/ct2/show/NCT02943824 (cited 2020 Apr 7). 